\title{
Force on a storage ring vacuum chamber after sudden turn-off of a magnet power supply
}

\author{
Gautam Sinha and S. S. Prabhu \\ AMTD, Raja Ramanna Centre for Advanced Technology, Indore-452013, India
}

(Received 5 July 2011; published 25 October 2011)

\begin{abstract}
We are commissioning a $2.5 \mathrm{GeV}$ synchrotron radiation source (SRS) where electrons travel in high vacuum inside the vacuum chambers made of aluminum alloys. These chambers are kept between the pole gaps of magnets and are made to facilitate the radiation coming out of the storage ring to the experimental station. These chambers are connected by metallic bellows. During the commissioning phase of the SRS, the metallic bellows became ruptured due to the frequent tripping of the dipole magnet power supply. The machine was down for quite some time. In the case of a power supply trip, the current in the magnets decays exponentially. It was observed experimentally that the fast $B$ field decay generates a large eddy current in the chambers and consequently the chambers are subjected to a huge Lorentz force. This motivated us to develop a theoretical model to study the force acting on a metallic plate when exposed to an exponentially decaying field and then to extend it for a rectangular vacuum chamber. The problem is formulated using Maxwell's equations and converted to the inhomogeneous Helmholtz equation. After taking the Laplace transform, the equation is solved with appropriate boundary conditions. Final results are obtained after taking the appropriate inverse Laplace transform. The expressions for eddy current contour and magnetic field produced by the eddy current are also derived. Variations of the force on chambers of different wall thickness due to spatially varying and exponentially time decaying field are presented. The result is a general theory which can be applied to different geometries and calculation of power loss as well. Comparisons are made with results obtained by simulation using a finite element based code, for quick verification of the theoretical model.
\end{abstract}

DOI: $10.1103 /$ PhysRevSTAB.14.102401

PACS numbers: 41.20.Gz, 07.30.Kf, 81.70.Ex

\section{INTRODUCTION}

A storage ring which produces synchrotron radiation is a type of circular particle accelerator in which a continuous or pulsed particle beam circulates in vacuum for several hours. It consists of many vacuum chambers where a charged particle beam travels under the influence of magnets positioned around the circumference of the ring. The dipole magnets define the orbit, the quadrupole magnets define the optics, and the sextupole magnets define the chromaticity. The required magnetic fields must be applied to keep the particles on the well-defined orbit. Therefore the vacuum chambers are placed between the poles of the magnets. If the magnets produce a time varying field then it will generate an eddy current in the vacuum chamber which in turn modifies the $B$ field produced by the accelerator magnets. That can cause disturbance to the circulating beam [1-3]. Beam position monitors (BPMs) are mounted at vacuum chambers to detect the position of the particle beam. The general profile of a storage ring dipole magnet vacuum chambers consists of the particle beam channel, the antechamber, and the slit (between the channel and the antechamber). The particle beam channel

Published by the American Physical Society under the terms of the Creative Commons Attribution 3.0 License. Further distribution of this work must maintain attribution to the author(s) and the published article's title, journal citation, and DOI. provides an ample aperture for the passage of the particle beam. The antechamber provides space for mounting photon absorbers at the top and/or bottom, leaving satisfactory clearance for the synchrotron radiation exit. The two parts are connected by a longitudinal slit, which allows the synchrotron radiation to leave the particle beam channel and should be sufficient for vacuum chamber pumping conductance [4-8].

The wall thickness of the chamber is decided to withstand the atmospheric pressure load when the chamber is under vacuum. In most of the cases, aluminum is chosen as the chamber material for its favorable vacuum, mechanical, and (non)magnetic properties. To get proper feedback of the beam, BPMs are installed on the chambers with very stringent mechanical tolerances and positional stability. To achieve this accuracy, the temperature of the tunnel is controlled within less than one degree Celsius. The vacuum chambers are supported at the BPM locations with Invar stands that have a low linear coefficient of thermal expansion [9].

In spite of all such precautions, one very important point which may have caused the disturbance of the positional accuracy of the BPM is overlooked. This point is the huge force acting on a chamber that arises due to the eddy current [1]. Eddy current will arise when the magnets are excited with harmonic or transient current. Harmonic excitations of magnets are required for boosters or rapid cycling synchrotrons, often used as injectors for large 
machines storing a charged particle circulating beam, and operated in the persistent mode, which are studied in detail and reported earlier by others including ourselves [1-3]. However, the effect of exponential decay of field has not been studied. It remains unexplored because it is not required for regular operation. However, in case of sudden switch off of the power supply, the current in the magnets will decay exponentially as in the case of L-R circuit. Crowbarring is done in a power supply circuit which includes a diode to allow the controlled current decay and avoid voltage spikes when the source is suddenly turned off [10].

We are commissioning a $2.5 \mathrm{GeV}$ synchrotron radiation source (SRS) where the electrons are injected at $550 \mathrm{MeV}$ and then the energy is increased to $2.5 \mathrm{GeV}$ in $300 \mathrm{~s}$ [11]. The electrons are traveling in high vacuum inside the vacuum chambers, made of aluminum alloys. These chambers are connected by metallic bellows. During the commissioning phase, the metallic bellows became ruptured due to the frequent tripping of the dipole magnet power supply. In case of power supply tripping, the current in the magnets decays exponentially. This generates large eddy current in the chambers and consequently the chambers are subjected to a huge force. This motivated us to develop an analytical model to study the force acting on a metallic plate when exposed to an exponentially decaying field and then to extend it for a rectangular vacuum chamber. This force should be considered at the design stage of a chamber and also for providing the support structure to achieve the required stringent positional accuracy of BPMs. Also the mechanical strength of the welding joints of a chamber should be strong enough to withstand the force acting on it when supported by rigid structure [12].

In this paper we have proposed a theoretical model to understand the eddy current behavior on a square/rectangular plate and the consequences of it. We describe the problem mathematically by Maxwell's equations and obtain a partial differential equation. It is assumed that the conducting plate is nonmagnetic. In the case of a booster or rapid cycling synchrotron vacuum chambers are placed between magnet poles where the field is mostly uniform and perpendicular to the surface of the chamber. But, in the case of a storage ring only the beam channel part of a chamber remains inside the dipole magnet poles and is exposed to a uniform $B$ field. The antechamber part remains outside the poles and is exposed to a space varying field. So, we have considered two different cases: (i) case I-the plate is exposed to a spatially uniform but exponentially time decaying field; and (ii) case II-the exponentially time decaying field is uniform on some portion of the plate but exponentially reducing with distance to the edge of the plate. In our earlier work we have considered the field of the form of $\cos (\omega t)$ and eddy current $\sin (\omega t)$. In the case of an exponential decay, the procedure is considerably different. Here, we have taken the Laplace transform and then solve the differential equation with appropriate boundary conditions. Final results are obtained after taking the inverse Laplace transform. The present model can provide accurately the following parameters: (i) eddy current contour in the plate; (ii) force acting on it; (iii) magnetic field produced by the induced eddy current. Comparisons are made with results obtained by simulation using a finite element based code, like OPERA $[13,14]$ for quick verification of the theoretical model.

\section{EXPERIMENTAL OBSERVATION}

Our 2.5 GeV Indus-2 (SRS) lattice, a double bend achromat, consists of eight super periods each having two dipole bending magnets, four focusing and five defocusing quadrupoles, and four sextupoles and seven corrector (or steering) magnets used for closed orbit correction [15]. The maximum field and the magnetic arclength of the dipole are $1.5 \mathrm{~T}$ and $2.17948 \mathrm{~m}$, respectively. The vacuum chambers of the Indus-2, made of aluminum alloy (5083-H321), are placed inside the $50 \mathrm{~mm}$ pole gap of the "C"-type dipole magnets. A large fraction of the total cross section of the vacuum chamber lies outside the pole gap of the magnet to facilitate the paths for synchrotron radiation (Fig. 1). During the commissioning phase it was noticed that some of the metallic bellows connecting the dipole chambers with the adjacent straight sections got damaged due to the movement of the dipole chambers.

We have seen from our earlier work when a conducting plate is exposed to a nonuniform time varying magnetic field it experiences unbalanced force [1]. The width of the chamber is about $0.646 \mathrm{~m}$. About $40 \%$ of it is covered by the poles of a dipole magnet and exposed to a uniform magnetic field. The remaining $60 \%$ of the chamber is lying

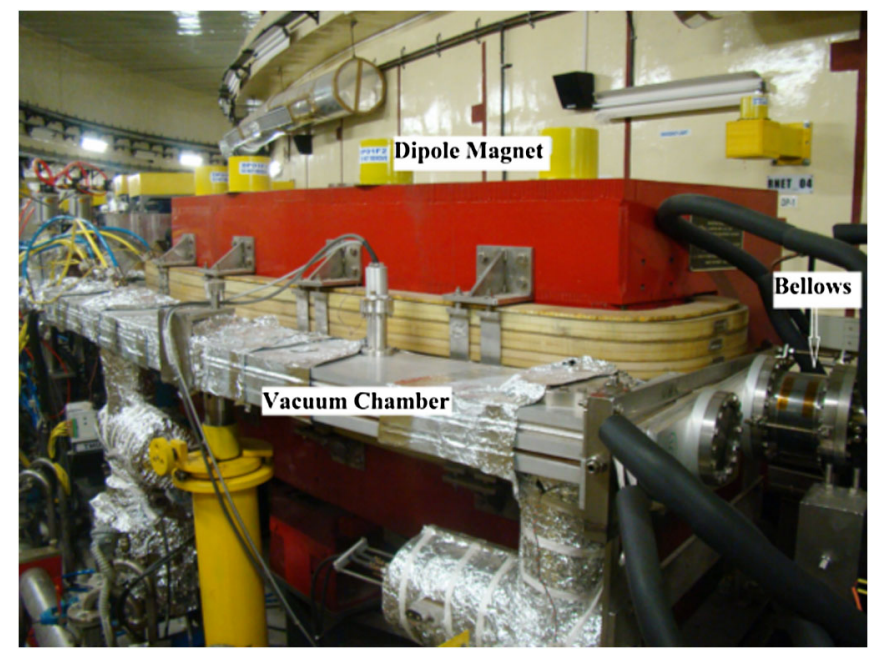

FIG. 1. The vacuum chamber made of aluminum alloy is placed inside the pole gap of a dipole magnet and approximately $60 \%$ of it is lying outside the pole gap and exposed to a nonuniform field. 

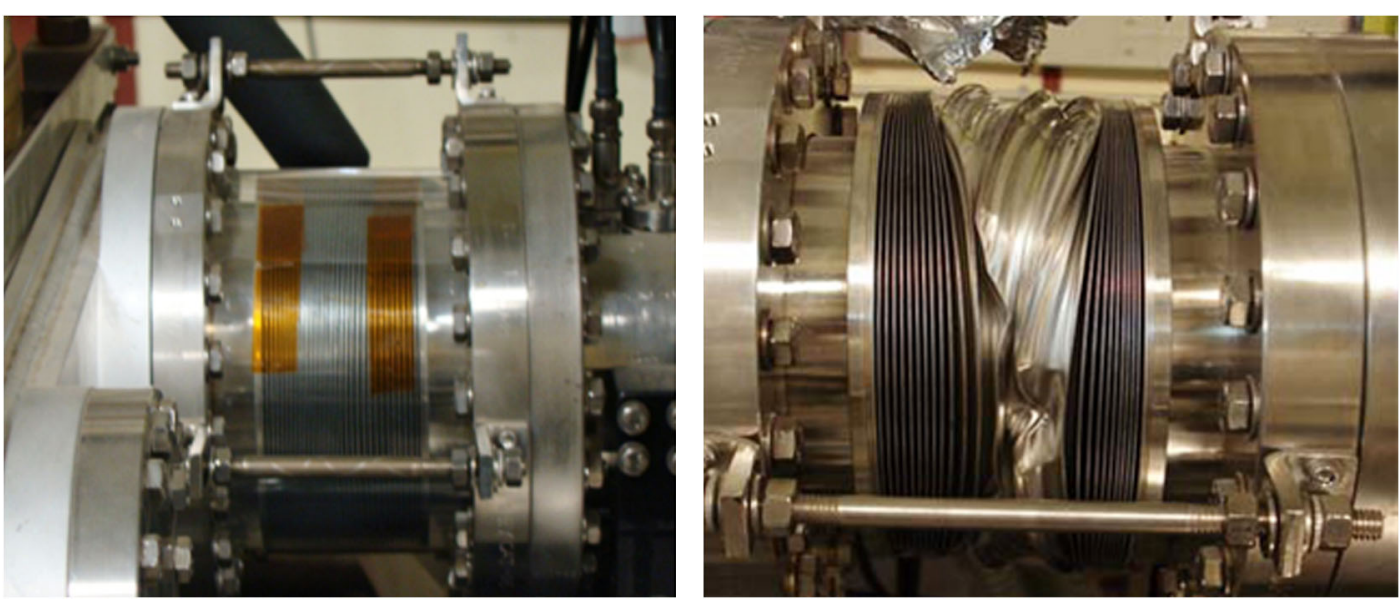

FIG. 2. The photograph of the bellow is shown before (left) and after (right) deformation.

outside the pole gap where the field is reducing exponentially with distance as one moves towards the edge of the chamber. The total length of the chamber is about $3 \mathrm{~m}$ and the typical weight of it is $300 \mathrm{~kg}$. In this storage ring beam is injected at $550 \mathrm{MeV}$ and then the energy is increased to $2.5 \mathrm{GeV}$ in $300 \mathrm{~s}$. In the case of normal operation eddy current generated in the chamber is negligible because of the slow ramp. However, due to some unavoidable reason, the dipole magnet power supply tripped several times during commissioning. The required peak field of the dipole magnet is $1.5 \mathrm{~T}$ which is obtained at $760 \mathrm{~A}$ current. The effective length of the dipole magnet is $2.17948 \mathrm{~m}$. The time constant of the power supply with all the loads connected is measured as $1.4 \mathrm{~s}$. So the current in the magnets decays as $I_{0} \exp (-t / 1.4)$, where $t$ is in s, after the tripping of the power supply that powered the dipole magnets. This fast decay of current and hence the field will generate a huge amount of eddy current in the chambers. Interaction of it with the dipole field caused around $18.38 \mathrm{kN}$ force acting on the chamber. To confirm this we purposefully switched off the power supply and captured the movement of the chamber and did some measurements. The metallic bellows got deformed due to the huge force. We have shown the deformed bellows in Fig. 2.

We have estimated the peak force using a 3D simulation as well [4]. Based on the analysis additional support structures are put in place to prevent any chamber movement. This work was performed and experimentally verified by our ultrahigh vacuum technology division. Apart from the bellows several other devices, like BPMs and pumps, are connected with the chambers whose alignments are also disturbed. We presume similar incidents may have occurred in other rings also but remain unnoticed because of its rare occurrence. We would also like to put a word of caution that during design stage the chamber and the support structure should be strong enough to withstand such forces to avoid any unwanted situation. To understand the physics behind it, we are trying to develop a theoretical model which will successfully explain all the above observations.

\section{ELECTROMAGNETIC FORMULATION OF THE THEORETICAL MODEL}

The actual shape of a dipole magnet vacuum chamber is a bit complicated. However, the eddy current depends on the flux changes and, hence, the perpendicular cross section of the chamber exposed to the changing field. Therefore, for eddy current calculation, we have simplified the geometry and considered a uniform wall thickness rectangular shape chamber. It consists of two horizontal plates connected by two thin vertical plates at the ends as shown in Figs. 3 and 4. Let us consider a conducting but nonmagnetic plate of width $a$, length $b$, and depth $d$ $(d \ll a$ and $b$ ), which is exposed to a time varying magnetic field directed along vertical $(Z)$ direction. The nature of the field variation is assumed to be transient like

$$
\vec{B}(x, y, t)=B_{0}(x, y) e^{-(t / \tau)} \hat{z} .
$$

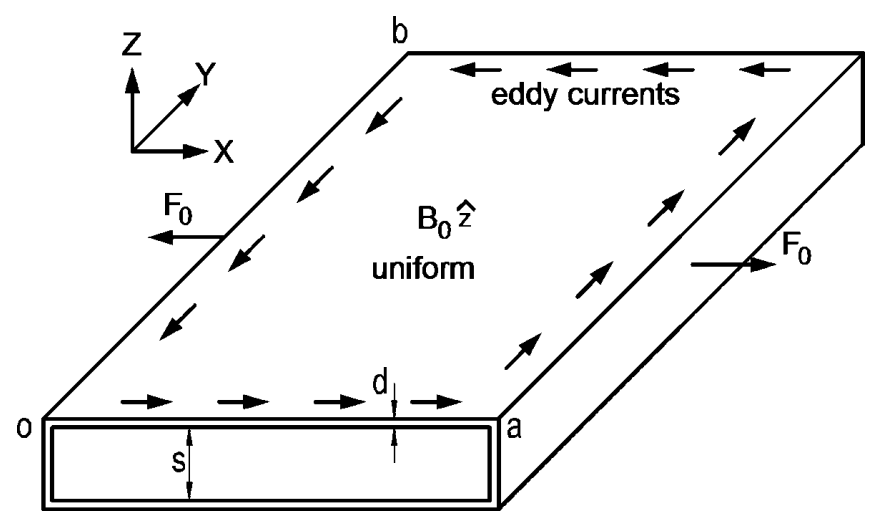

FIG. 3. Coordinate system, geometry of the chamber and the direction of eddy current and the force $\left(F_{0}\right)$ is shown when the chamber is exposed to a spatially uniform but exponentially time decaying magnetic field, $B_{0}$ (case I). 


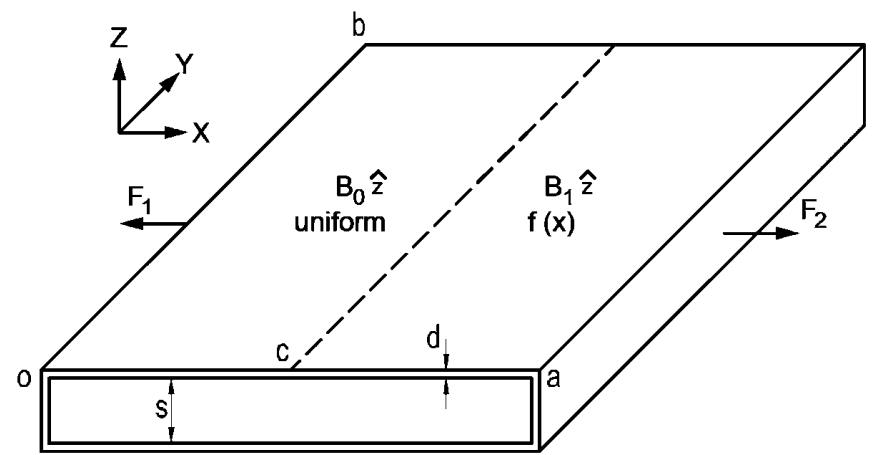

FIG. 4. Coordinate system, geometry of the chamber and the direction of the forces $\left(F_{1}\right.$ and $\left.F_{2}\right)$ is shown. Here the chamber is exposed to a magnetic field that varies both in space and time (case II).

We consider the following spatial variations of the magnetic field, $B_{0}(x, y)$ :

Case I: $B_{0}(x, y)=B_{0}$ for $0 \leq x \leq a, 0 \leq y \leq b$

Case II: $B_{0}(x, y)=B_{0}$ for $0 \leq x \leq c, 0 \leq y \leq b$

$$
=B_{0} e^{-(x-c) / \lambda} \text { for } c \leq x \leq a, 0 \leq y \leq b,
$$

where $c<a$. Hereafter we will refer these as case I and case II, respectively.

The Maxwell's equations in the absence of the plate can be written as

$$
\vec{\nabla} \times \vec{B}=\mu_{0} \varepsilon_{0} \frac{\partial \vec{E}}{\partial t} \quad \vec{\nabla} \times \vec{E}=-\frac{\partial \vec{B}}{\partial t},
$$

where $\vec{E}$ is the electric field, $\mu_{0}$ is the permeability, and $\varepsilon_{0}$ is the dielectric constant of free space. The time varying magnetic field will induce an eddy current in the conducting plate. The eddy current will produce an induced field, $\vec{H}_{i}$, that will oppose the applied field. So, the Maxwell's equations in the presence of the plate can be written as

$$
\begin{aligned}
\vec{\nabla} \times\left(\vec{B}+\mu_{0} \vec{H}_{i}\right) & =\mu_{0} \vec{J}+\mu_{0} \varepsilon_{0} \frac{\partial \vec{E}}{\partial t} \\
\vec{\nabla} \times \vec{E} & =-\frac{\partial\left(\vec{B}+\mu_{0} \vec{H}_{i}\right)}{\partial t},
\end{aligned}
$$

where $\vec{J}$ is the current density in the plate. Using Eqs. (3) and (4) we find

$$
\vec{\nabla} \times \vec{H}_{i}=\vec{J} .
$$

Eddy current flows in a closed loop, in other words, $\vec{\nabla}$. $\vec{J}=0$. Therefore, $\vec{J}$ can be written as the curl of a potential function $u(x, y, t)$ :

$$
\vec{J}=\frac{1}{d} \vec{\nabla} \times\{u(x, y, t) \hat{z}\} .
$$

Using Eqs. (5) and (6) we obtain

$$
\begin{aligned}
\vec{\nabla} \times\left\{\vec{H}_{i}-\frac{u(x, y, t)}{d} \hat{z}\right\} & =0 \\
\text { or }\left\{\vec{H}_{i}-\frac{u(x, y, t)}{d} \hat{z}\right\} & =\vec{\nabla} V(x, y, z, t) \\
\text { or } \vec{H}_{i} & =\left\{\frac{u(x, y, t)}{d} \hat{z}+\vec{\nabla} V(x, y, z, t)\right\},
\end{aligned}
$$

where $V(x, y, z, t)$ is a scalar magnetic potential. In the present model the eddy current is flowing on the surface of the plate, i.e. $j_{z}=0$. We are interested in finding the eddy current distribution on the plate. The $Z$ component of the field will depend on $\partial V(x, y, z, t) / \partial z$ which should be a function of $x$ and $y$ and time on the surface of the plate. Therefore, we have presumed that $\partial V(x, y, z, t) / \partial z$ will be related to a general function $u(x, y, t)$, already assumed. So, without loss of generality, we assumed the induced field along the $Z$ direction to be

$$
H_{i}^{z}=\left\{\frac{u(x, y, t)}{d}+g u(x, y, t)\right\}=(1+g d) \frac{1}{d} u(x, y, t),
$$

where $g$ is constant and $H_{i}^{z}$ is evaluated on the surface of the metallic plate. Using Ohm's law $\vec{J}=\sigma \bar{E}$, we find from Eq. (4)

$$
\begin{aligned}
\left\{\frac{\partial J_{y}}{\partial x}-\frac{\partial J_{x}}{\partial y}\right\} \hat{z}= & -\sigma\left\{-\frac{B_{0}(x, y)}{\tau} e^{-(t / \tau)}\right. \\
& \left.+\mu_{0} \frac{(1+g d)}{d} \frac{\partial u(x, y, t)}{\partial t}\right\} \hat{z} .
\end{aligned}
$$

Substituting the expression of $\vec{J}$ from Eq. (6), we obtain

$$
\begin{gathered}
\left(\frac{\partial^{2}}{\partial x^{2}}+\frac{\partial^{2}}{\partial y^{2}}\right) u(x, y, t)-\mu_{0} \sigma(1+g d) \frac{\partial u(x, y, t)}{\partial t} \\
=-\frac{\sigma B_{0}(x, y) d}{\tau} e^{-(t / \tau)},
\end{gathered}
$$

where $\sigma$ is the conductivity of the plate. The above inhomogeneous Helmholtz equation can be solved with certain boundary conditions to obtain $u(x, y, t)$ and, hence, the eddy current contour in the plate.

\section{A. BOUNDARY VALUE PROBLEM AND EDDY CURRENT}

We now solve Eq. (10) in two specific cases as given in Eq. (2). The relevant boundary conditions are given by

$u(x, y, 0)=0 \quad$ for $t>0$

$u(0, y, t)=u(a, y, t)=u(x, 0, t)=u(x, b, t)=0$.

Let the Laplace transform of $u(x, y, t)$ be $u(x, y, s)$, then we can write

$$
u(x, y, s)=L\{u(x, y, t)\}=\int_{0}^{\infty} e^{-s t} u(x, y, t) \partial t .
$$

Multiplying Eq. (10) by $e^{-s t}$ and integrating over $d t$ we get 


$$
\begin{gathered}
\left(\frac{\partial^{2}}{\partial x^{2}}+\frac{\partial^{2}}{\partial y^{2}}\right) u(x, y, s)-\mu_{0} \sigma(1+g d) s u(x, y, s) \\
=-\frac{\sigma B_{0}(x, y) d}{1+s \tau}
\end{gathered}
$$

Here, we have used the identities

$$
\begin{gathered}
\int_{0}^{\infty} e^{-s t} \frac{\partial u(x, y, t)}{\partial t} \partial t=s u(x, y, s) \quad \text { and } \\
\int_{0}^{\infty} e^{-s t} e^{-(t / \tau)} \partial t=\frac{\tau}{1+s \tau}
\end{gathered}
$$

The eigenfunction $u_{n m}(x, y, s)$ of the equation

$$
\left(\frac{\partial^{2}}{\partial x^{2}}+\frac{\partial^{2}}{\partial y^{2}}\right) u_{n m}(x, y, s)=\lambda_{n m} u_{n m}(x, y, s)
$$

with the above boundary conditions can then be written as

$$
u_{n m}(x, y, s)=N_{n m} \sin \left(\nu_{n} x\right) \sin \left(\mu_{m} y\right)
$$

where $\nu_{n}=\frac{n \pi}{a}, \mu_{m}=\frac{m \pi}{b}$ ( $n, m$ are integers), and $N_{n m}=$ $\frac{2}{\sqrt{a b}}$ are obtained using a normalization condition.

The general solutions $u^{I}(x, y, s)$ and $u^{I I}(x, y, s)$ of Eq. (12) for the two cases as mentioned in Eq. (2) may then be expressed as

$$
\begin{aligned}
& u^{I}(x, y, s)=\sum_{n, m=1}^{\infty} A_{n m}^{I}(s) N_{n m} \sin \left(\nu_{n} x\right) \sin \left(\mu_{m} y\right) \\
& u^{I I}(x, y, s)=\sum_{n, m=1}^{\infty} A_{n m}^{I I}(s) N_{n m} \sin \left(\nu_{n} x\right) \sin \left(\mu_{m} y\right),
\end{aligned}
$$

where the value of the $A_{n m}$ can be obtained as follows. Put the value of $u(x, y, s)$ in Eq. (12) and use the orthogonality condition $\int_{0}^{a} \partial x \int_{0}^{b} \partial y u_{n m}(x, y) u_{n^{\prime} m^{\prime}}(x, y)=\delta_{n n^{\prime}} \delta_{m m^{\prime}}$. After rearrangement we get

$$
\begin{array}{r}
A_{n m}^{I}(s)=\frac{2 \sigma B_{0} d}{\sqrt{a b}(1+s \tau)}\left\{\frac{K_{n m}^{0}(a, b)}{\left(\nu_{n}^{2}+\mu_{m}^{2}\right)+\sigma \mu_{0}(1+g d) s}\right\} \\
\text { and } A_{n m}^{I I}(s)=\frac{2 \sigma B_{0} d}{\sqrt{a b}(1+s \tau)}\left\{\frac{K_{n m}(a, b, c)}{\left(\nu_{n}^{2}+\mu_{m}^{2}\right)+\sigma \mu_{0}(1+g d)_{s}}\right\}
\end{array}
$$

with

$$
\begin{aligned}
K_{n m}^{0}(a, b) & =\int_{0}^{a} \sin \nu_{n} x \partial x \int_{0}^{b} \sin \mu_{m} y \partial y \\
& =\frac{\left\{1-\cos \left(\nu_{n} a\right)\right\}\left\{1-\cos \left(\mu_{m} b\right)\right\}}{\nu_{n} \mu_{m}}
\end{aligned}
$$

and

$$
\begin{aligned}
K_{n m}(a, b, c)= & \left\{\int_{0}^{c} \sin \nu_{n} x \partial x+\int_{c}^{a} e^{-(x / \lambda)} e^{c / \lambda} \sin \nu_{n} x \partial x\right\} \\
& \times \int_{0}^{b} \sin \mu_{m} y \partial y \\
= & \left(1-\frac{\lambda^{2} \nu_{n}^{2}}{1+\lambda^{2} \nu_{n}^{2}}\right) K_{n m}^{0}(c, b) \\
& +\left(\frac{\lambda^{2} \nu_{n}^{2}}{1+\lambda^{2} \nu_{n}^{2}}\right) e^{(c-a) / \lambda} K_{n m}^{0}(a, b) \\
& +\left(\frac{\lambda^{2} \nu_{n}^{2}}{1+\lambda^{2} \nu_{n}^{2}}\right) L_{n m}^{0}(c, b) \\
& -\left(\frac{\lambda^{2} \nu_{n}^{2}}{1+\lambda^{2} \nu_{n}^{2}}\right) e^{(c-a) / \lambda} L_{n m}^{0}(a, b) .
\end{aligned}
$$

Here $\quad L_{n m}^{0}(a, b)=\left[\left\{1-\cos \left(\mu_{m} b\right)\right\} /\left(\nu_{n} \mu_{m}\right)\right]\left\{1+\frac{\sin \left(\nu_{n} a\right)}{\lambda \nu_{n}}\right\}$ and $L_{n m}^{0}(c, b)$ can be obtained by replacing $a$ by $c$.

We make use of the following integration to get the above expression:

$$
\begin{aligned}
I= & \int_{c}^{a} e^{c / \lambda} e^{-x / \lambda} \sin \left(\nu_{n} x\right) \partial x \\
= & \frac{1}{\left(1+\frac{1}{\lambda^{2} \nu_{n}^{2}}\right)}\left[-\frac{e^{(c-a) / \lambda}}{\nu_{n}}\left\{\cos \left(\nu_{n} a\right)+\frac{\sin \left(\nu_{n} a\right)}{\lambda \nu_{n}}\right\}\right. \\
& \left.+\frac{1}{\nu_{n}}\left\{\cos \left(\nu_{n} c\right)+\frac{\sin \left(\nu_{n} c\right)}{\lambda \nu_{n}}\right\}\right]
\end{aligned}
$$

Now, to get the value of $u(x, y, t)$ from $u(x, y, s)$, we have to take the inverse Laplace transform:

$$
\begin{aligned}
u(x, y, t) & =L^{-1}\{u(x, y, s)\} \\
& =\sum_{n, m=1}^{\infty} L^{-1}\left\{A_{n m}^{I}(s)\right\} N_{n m} \sin \left(\nu_{n} x\right) \sin \left(\mu_{m} y\right) \\
& =\sum_{n, m=1}^{\infty} A^{I}{ }_{n m}(t) N_{n m} \sin \left(\nu_{n} x\right) \sin \left(\mu_{m} y\right)
\end{aligned}
$$

Rearranging the expression of Eq. (16a), we get

$$
A_{n m}^{I}(s)=\frac{2 B_{0} d K_{n m}^{0}(a, b)}{\sqrt{a b} \mu_{0}(1+g d) \tau\left\{s+\frac{1}{\tau}\right\}\left\{s+\frac{\nu_{n}^{2}+\mu_{m}^{2}}{\sigma \mu_{0}(1+g d)}\right\}} .
$$

Taking the inverse Laplace transform, we get

$$
\begin{aligned}
A_{n m}^{I}(t)= & \frac{2 B_{0} d K_{n m}^{0}(a, b)}{\sqrt{a b} \mu_{0}(1+g d) \tau\left\{\frac{\nu_{n}^{2}+\mu_{m}^{2}}{\sigma \mu_{0}(1+g d)}-\frac{1}{\tau}\right\}} \\
& \times\left[e^{-(t / \tau)}-e^{-t\left\{\left(\nu_{n}^{2}+\mu_{m}^{2}\right) /\left(\sigma \mu_{0}(1+g d)\right)\right\}}\right] \\
A_{n m}^{I I}(t)= & \frac{2 B_{0} d K_{n m}(a, b, c)}{\sqrt{a b} \mu_{0}(1+g d) \tau\left\{\frac{\nu_{n}^{2}+\mu_{m}^{2}}{\sigma \mu_{0}(1+g d)}-\frac{1}{\tau}\right\}} \\
& \times\left[e^{-(t / \tau)}-e^{-t\left\{\left(\nu_{n}^{2}+\mu_{m}^{2}\right) /\left(\sigma \mu_{0}(1+g d)\right)\right\}}\right] .
\end{aligned}
$$


Here we have used the following identity [16]:

$$
L^{-1}\left\{\frac{1}{(s+p)(s+q)}\right\}=\frac{e^{-p t}-e^{-q t}}{q-p}, \quad p \neq q .
$$

Therefore, the eddy current density and the induced field due to the eddy current on the surface of the plate can be expressed as

$$
\begin{aligned}
j_{x}= & \frac{1}{d} \frac{\partial u(x, y, t)}{\partial y}=\sum_{n, m=1}^{\infty} \frac{\mu_{m}}{d} A_{\mathrm{nm}}^{\alpha}(t) N_{n m} \sin \left(\nu_{n} x\right) \cos \left(\mu_{m} y\right) \\
j_{y}= & -\frac{1}{d} \frac{\partial u(x, y, t)}{\partial x}=-\sum_{n, m=1}^{\infty} \frac{\nu_{n}}{d} A_{\mathrm{nm}}^{\alpha}(t) N_{n m} \cos \left(\nu_{n} x\right) \\
& \times \sin \left(\mu_{m} y\right) \\
H_{i}^{z}= & \frac{1}{d}(1+g d) u(x, y, t) \\
= & \sum_{n, m=1}^{\infty} \frac{1}{d}(1+g d) A_{n m}^{\alpha}(t) N_{n m} \sin \left(\nu_{n} x\right) \sin \left(\mu_{m} y\right),
\end{aligned}
$$

where $\alpha$ represents $I$ or $I I$. It is clear from Eq. (18) that the time decay of eddy current and field produced by it will follow a new time constant $\tau_{1}$ which is different from the time constant of the applied field decay, $\tau$. The new time constant will depend on the geometry and the conductivity of the plate.

It is clear from Eqs. (18) and (19) that the value of the constant " $g$ " needs to be evaluated in order to calculate the values of current density and field. The value of the constant $g$ can be determined self-consistently as explained below. The value of $H_{i}^{z}$ is evaluated on the surface of the plate using Eq. (19) and is proportional to $(1+g d)$. By knowing the values of $j_{x}$ and $j_{y}$, the $B$ field on the surface of the plate can also be evaluated using Biot-Savart's law which also depends on $(1+g d)$ [see Sec. III C, Eq. (27)]. Now, we plot the $B$ field obtained using two different

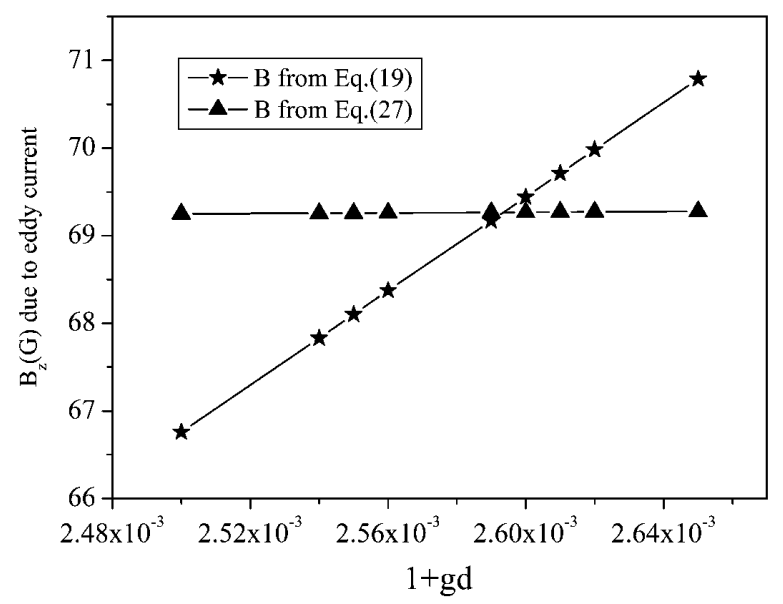

FIG. 5. Plot of the magnetic field on the surface of the plate obtained using two different methods using Eqs. (19) and (27), respectively. The value of $(1+g d)$ is obtained from the intersection of two curves. methods as explained above against $(1+g d)$. The value of $(1+g d)$ is obtained from the intersection of the above two curves plotted in a graph (Fig. 5). Finally, the value of $g$ is obtained by using the value of $d$ which is the thickness of the plate. While evaluating Eq. (19) we have taken the values of $m$ and $n$, the index of sum, up to 300 because beyond this limit the change in current density is less than $0.002 \%$. So, instead of evaluating an infinite series sum, the sum of index up to 300 is sufficient to calculate the values of current density and field without losing much accuracy.

Now, we will use the eddy current expression to find the Lorentz force acting on the plate in the presence of an applied magnetic field.

\section{B. LORENTZ FORCE}

If an eddy current flows through the conducting plate, then it will experience a force in the presence of the magnetic field. The $B$ field is uniform throughout the plate. The amplitude of the eddy current increases symmetrically as we move away from the center of the plate. But, the sign of the eddy current $j_{y}\left(j_{x}\right)$ is positive on the right (lower) side of the plate and negative on the on the left (upper) side (Figs. 8 and 7). So, the resulting force on one half of the plate will be balanced by the other half and there will be no resultant force acting on the plate (case I). However, this balance will be disturbed when the plate is exposed to a nonuniform field (case II). It is very important for the stability of any system to estimate the magnitude of the force. The force can be expressed as

$$
\begin{aligned}
\vec{F}= & \int_{V}(\vec{J} \times \vec{B}) \partial V \\
= & B_{0} e^{-(t / \tau)} d\left\{\int_{0}^{a} \partial x\right\}\left\{\int_{0}^{b}\left(j_{y} \hat{x}-j_{x} \hat{y}\right) \partial y\right\} \quad \text { case I } \\
= & B_{0} e^{-(t / \tau)} d\left\{\int_{0}^{c} \partial x+\int_{c}^{a} e^{-(x-c) / \lambda} \partial x\right\} \\
& \times\left\{\int_{0}^{b}\left(j_{y} \hat{x}-j_{x} \hat{y}\right) \partial y\right\} \quad \text { case II. }
\end{aligned}
$$

Substituting $\vec{J}$ from Eq. (19) to Eq. (20), the net force acting on the plate can be calculated. In case I,

$$
\begin{aligned}
& \vec{F}_{Y}=0 \text { because } \int_{o}^{b} \cos \left(\mu_{m} y\right) \partial y=0 \\
& \vec{F}_{X}=0 \text { because } \int_{o}^{a} \cos \left(\nu_{n} x\right) \partial x=0 .
\end{aligned}
$$

In case II, there will be a net force acting on the plate:

$$
\vec{F}_{Y}=0 \text { because } \int_{o}^{b} \cos \left(\mu_{m} y\right) \partial y=0
$$

$$
\begin{aligned}
\vec{F}_{X}= & -\left[B _ { 0 } e ^ { - ( t / \tau ) } d \sum _ { n , m = 1 } ^ { \infty } \frac { \nu _ { n } } { d } A _ { n m } ^ { I I } ( t ) N _ { n m } \left\{\int_{0}^{c} \partial x\right.\right. \\
& \left.\left.+\int_{c}^{a} e^{-(x-c) / \lambda} \partial x\right\} \cos \left(\nu_{n} x\right) \int_{0}^{b} \sin \left(\mu_{m} y\right) \partial y\right] \hat{x} .
\end{aligned}
$$


After integration and rearranging we get

$$
\begin{aligned}
\vec{F}_{X}= & -B_{0} e^{-(t / \tau)} \sum_{n, m=1}^{\infty} \frac{1}{\mu_{m}} A_{n m}^{I I}(t) N_{n m}\left[1-\cos \left(\mu_{m} b\right)\right] \\
& \times\left[\sin \left(\nu_{n} c\right)+\left(\frac{\lambda^{2} \nu_{n}^{2}}{1+\lambda^{2} \nu_{n}^{2}}\right)\left[\begin{array}{c}
e^{(c-a) / \lambda}\left\{\sin \left(\nu_{n} a\right)-\frac{1}{\lambda \nu_{n}} \cos \left(\nu_{n} a\right)\right\} \\
-\left\{\sin \left(\nu_{n} c\right)-\frac{1}{\lambda \nu_{n}} \cos \left(\nu_{n} c\right)\right\}
\end{array}\right]\right] \hat{x} .
\end{aligned}
$$

The final expression is obtained by using the following integrals:

$$
\begin{aligned}
I & =\int_{c}^{a} e^{-x / \lambda} \cos \left(\nu_{n} x\right) \partial x=\left.e^{-x / \lambda} \frac{\sin \left(\nu_{n} x\right)}{\nu_{n}}\right|_{c} ^{a}+\frac{1}{\lambda \nu_{n}} \int_{c}^{a} e^{-x / \lambda} \frac{\sin \left(\nu_{n} x\right)}{\nu_{n}} \partial x \\
& =\left.\left(e^{-x / \lambda} \frac{\sin \left(\nu_{n} x\right)}{\nu_{n}}\right)\right|_{c} ^{a}+\frac{1}{\lambda \nu_{n}}\left\{\left.\left(-e^{-x / \lambda} \frac{\cos \left(\nu_{n} x\right)}{\nu_{n}}\right)\right|_{c} ^{a}-\frac{1}{\lambda \nu_{n}} \int_{c}^{a} e^{-x / \lambda} \cos \left(\nu_{n} x\right) \partial x\right\} \\
\text { or } I e^{c / \lambda} & =\frac{1}{\nu_{n}}\left(\frac{\lambda^{2} \nu_{n}^{2}}{1+\lambda^{2} \nu_{n}^{2}}\right)\left[e^{(c-a) / \lambda}\left\{\sin \left(\nu_{n} a\right)-\frac{1}{\lambda \nu_{n}} \cos \left(\nu_{n} a\right)\right\}-\left\{\sin \left(\nu_{n} c\right)-\frac{1}{\lambda \nu_{n}} \cos \left(\nu_{n} c\right)\right\}\right] .
\end{aligned}
$$

As the force depends on the eddy current, we have taken the sum of $m$ and $n$ up to 300 only without losing too much accuracy.

\section{MAGNETIC FIELD PRODUCED BY EDDY CURRENT}

We have already calculated the eddy current distribution in the conducting plate induced by a time varying magnetic field. We have assumed that the thickness of the plate is uniform and quite small compared to the skin depth. This assumption may be fine for the vacuum chambers used in accelerators which produce synchrotron radiation. The source current density for which the induced field needs to be calculated is lying on the $x-y$ plane. The eddy current is distributed in the plate. We consider a small strip of the plate as a current carrying conductor. The magnetic field at a point $P(x, y, z)$ due to a source current at $\left(x^{\prime}, y^{\prime}, z^{\prime}\right)$ can be expressed using the Biot-Savart's law:

$$
\vec{B}(x, y, z)=\frac{\mu_{0}}{4 \pi} \int_{V} \frac{\vec{j} \times\left(\vec{r}-\vec{r}^{\prime}\right)}{\left(r-r^{\prime}\right)^{3}} \partial x^{\prime} \partial y^{\prime} \partial z^{\prime},
$$

where $r-r^{\prime}=\left(x-x^{\prime}, y-y^{\prime}, z-z^{\prime}\right)$.

By taking $z^{\prime}=0$ and taking the curl, we get

$$
\vec{B}_{0}(x, y, z)=\frac{d \mu_{0}}{4 \pi} \int_{0}^{a} \partial x^{\prime} \int_{0}^{b} \partial y^{\prime}\left[\begin{array}{c}
\frac{j_{x}\left(y-y^{\prime}\right)-j_{y}\left(x-x^{\prime}\right)}{\left\{\left(x-x^{\prime}\right)^{2}+\left(y-y^{\prime}\right)^{2}+z^{2}\right\}^{3 / 2}} \hat{z}+ \\
\frac{\left(-j_{x} z\right)}{\left\{\left(x-x^{\prime}\right)^{2}+\left(y-y^{\prime}\right)^{2}+z^{2}\right\}^{3 / 2}} \hat{y}+ \\
\frac{j_{y} z}{\left\{\left(x-x^{\prime}\right)^{2}+\left(y-y^{\prime}\right)^{2}+z^{2}\right\}^{3 / 2}} \hat{x}
\end{array}\right] .
$$

The above expression is true in the absence of any magnetic material. The field due to the eddy current is obtained after integrating the above expression. We have written a code in FORTRAN to evaluate the field. It is possible to calculate the field when the current carrying conductors are located between the two parallel iron poles with very high permeability. Details of it are available in our earlier work [1].

\section{RESULTS AND DISCUSSIONS}

To test the theoretical model we have calculated the eddy current generated in the two parallel aluminum plates when exposed to a spatially uniform but exponentially time decaying field. Direction of the field is perpendicular to the surface of both plates. The distance between the two plates $(s)$ is $20 \mathrm{~mm}$. The width $(a)$, length $(b)$, and thickness $(d)$ of the plates are 1.4, 1.4, and $0.002 \mathrm{~m}$, respectively. The conductivity of both plates is $16.95 \times 10^{6} \mathrm{~S} / \mathrm{m}$. The peak field and the time constant of the field decay are $1.3695 \mathrm{~T}$ and $1.4 \mathrm{~s}$, respectively. So, the transient field can be expressed as

$$
B=1.3965 e^{-(t / 1.4)},
$$

where $t$ is expressed in s. Before computing the eddy current density using Eq. (19), the value of $g$ and the index of sum ( $m$ and $n$ ) should be evaluated. We have plotted the value of field obtained on the surface of the plate using Eqs. (19) and (27) in Fig. 5. From the intersection of both curves we find that the value of $(1+g d)$ is $0.00259 \mathrm{~m}$. Therefore, the value of $g$ is -498.705 for the above geometry.

The values of the current density and the field in Eq. (19) are expressed in terms of an infinite series sum. However, the index of the sum should be limited to a reasonable small number to save the computation time without losing the accuracy. To estimate the necessary minimum index up to which the sum is computed, we have calculated the current density for various indices and plotted them in Fig. 6. It is clear from the figure that the index can be taken as 300 for calculation of different parameters. Variation of current density is found to be around $0.002 \%$ if the index of the sum is increased from 300 to 400 . Therefore, for all 


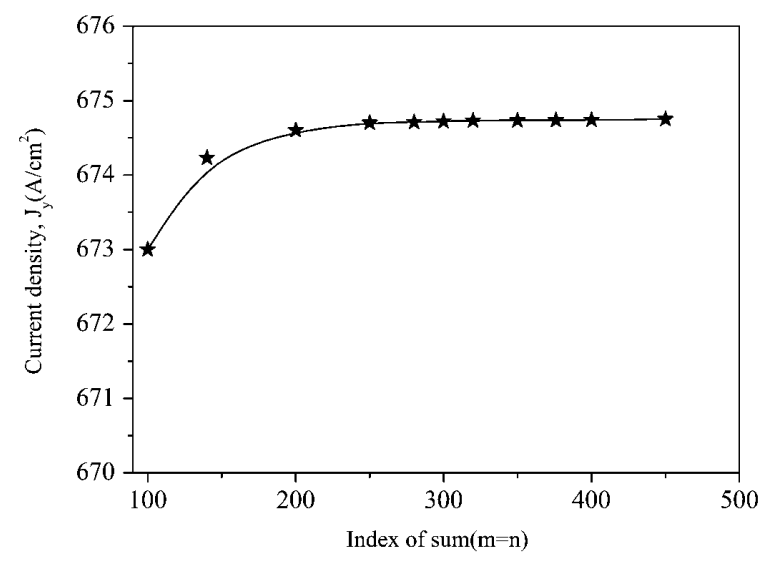

FIG. 6. Evaluation of the eddy current from the analytical expression of Eq. (19) for different indices of the sum $(m=n)$. The solid line is just a guide to the eye.

practical calculations, the index of the sum can be taken as 300. The data plotted in Fig. 5 is also obtained for $m=$ $n=300$.

Once the value of $g$ and the sum of indices $m$ and $n$ are known, current densities can be computed using the analytical expression given in Eq. (19). We have developed a code in FORTRAN for computation of eddy current and other required parameters. This code is compiled on a HP DL 380 G5 server having a xeon $3.73 \mathrm{GHz}$ dual core with 8GB RAM and Red Hat Enterprise Linux 4.0. The code takes typically a minute to calculate the value of current densities in the above-mentioned plate. The contour plot of current density $j_{x}$ and $j_{y}$ are plotted in Figs. 7 and 8, respectively.

Both contour plots are obtained when an aluminum plate of dimension $(1.4,1.4,0.002) \mathrm{m}^{3}$ is exposed to a spatially uniform but exponentially time decaying field of the form given in Eq. (28) and at time $t=0.2 \mathrm{~s}$. The $B$ field is

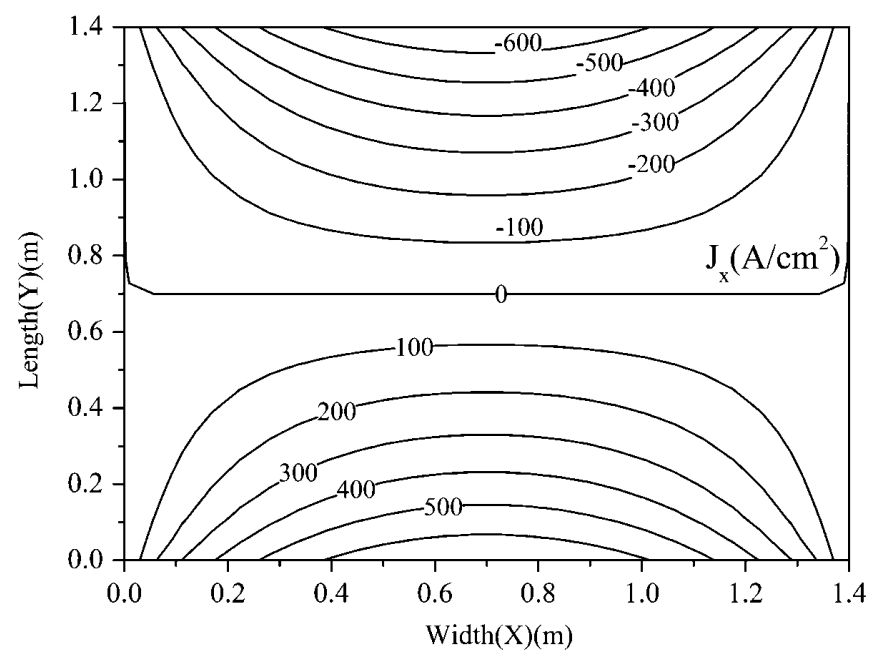

FIG. 7. Contour plot of $X$ component of the eddy current density, $j_{x}$, in the aluminum plate of dimension $(1.4,1.4,0.002) \mathrm{m}^{3}$.

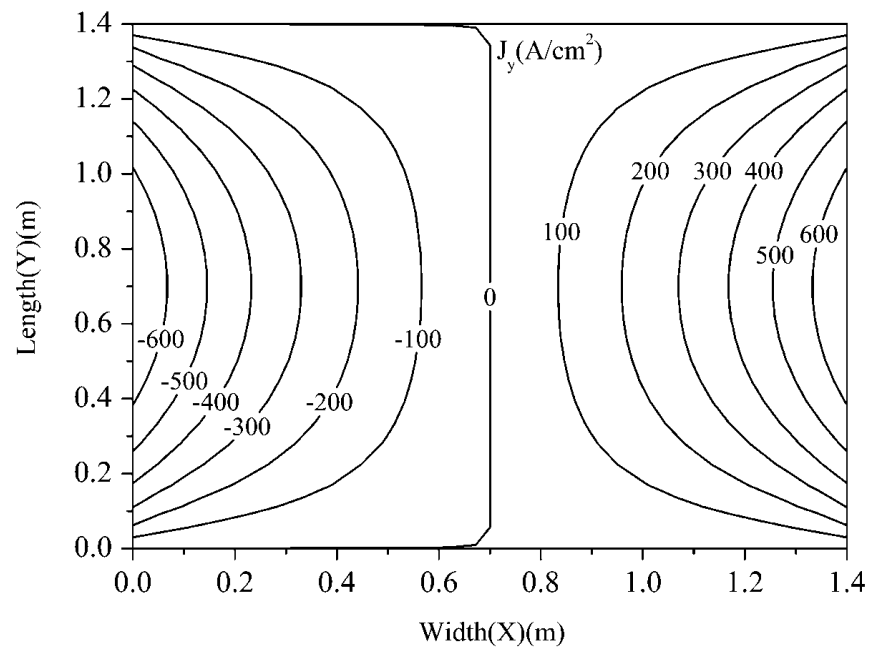

FIG. 8. Contour plot of $Y$ component of the eddy current density, $j_{y}$, in the aluminum plate of dimension $(1.4,1.4$, $0.002) \mathrm{m}^{3}$ when exposed to a field exponentially decaying with time.

pointing in the upward direction and decreasing with time. Therefore, the direction of the eddy current will be such that it will oppose the change of the field in accordance with the Lenz's law. So, the flow of eddy current will be in the counterclockwise direction. This phenomenon is depicted in Figs. 7 and 8 and has come naturally from the mathematical formulation of the model. The value of $j_{x}$ is negative and positive for the upper and lower half of the plate, respectively. The value of $j_{x}$ is the maximum at the center of the lower half and it passes through zero at the middle and becomes the minimum at the center of the upper half. It is symmetric about the line $Y=b / 2$. It is clear from Fig. 8 that the variation of $j_{y}$ is symmetric about the line $X=a / 2$. The value of $j_{y}$ is the maximum at

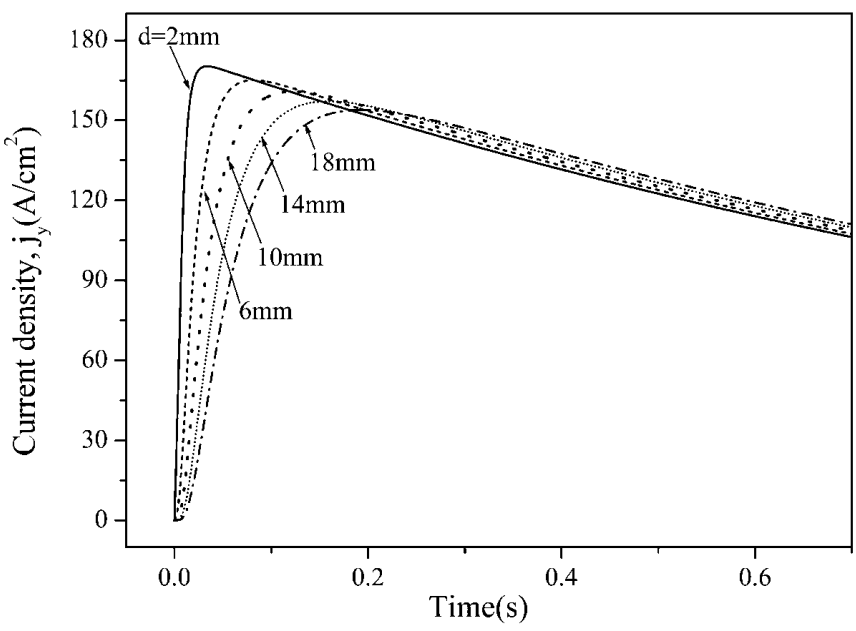

FIG. 9. Variation of the eddy current density, $j_{y}$, with time for various thicknesses $(d=2,6,10,14$, and $18 \mathrm{~mm}$, respectively) of a plate while the other dimensions are kept constant $(a=1.4 \mathrm{~m}$ and $b=1.4 \mathrm{~m})$. 
the center of the right edge as expected and it passes through zero at the line bisecting the plate along the width and the minimum is at the center of the left edge.

Variation of the current density with time shows interesting behavior. An electromotive force (emf) is generated across the plate due to the change of flux. This emf will drive the eddy current to flow in the plate in a closed path. As it is circulating in the plate an effective inductance will come into the picture. Time $t=0$ is taken at a time when the field starts decaying. So, at $t=0$ current density is also zero. Now, it will take finite time to reach to the peak value and then start decaying as the rate of change of flux is reducing with time. The effective inductance of a plate depends on the geometry of the plate. Resistance of the plate decreases with the increase of the thickness. Therefore, the time constant, which is the ratio of the inductance to the resistance, increases with the increase of thickness of a plate. So, the eddy current will take longer time to reach the peak value as the thickness of a plate increases. This is apparent from our theoretical model, Eqs. (18) and (19). The variation of the $Y$ component of the current density with time is plotted for different thickness $(2,6,10,14$, and $18 \mathrm{~mm})$ of a plate having both the width and length $1.4 \mathrm{~m}$ in Fig. 9. The time constant of the field decay is the same as $1.4 \mathrm{~s}$ for all the cases and $j_{y}$ is calculated at $X=0.9 \mathrm{~m}$ from the edge on the $Y=b / 2$ line. It shows that for a $2 \mathrm{~mm}$ thick plate, $j_{y}$ reaches very quickly $(0.02 \mathrm{~s})$ to the peak value. However, for the $18 \mathrm{~mm}$ thick plate it takes $0.19 \mathrm{~s}$ to reach the peak value.

Time variation of the current density at various points ( $X=0.8,1.0,1.2$, and $1.39 \mathrm{~m}$ from the edge) of a plate of dimension $(1.4,1.4,0.002) \mathrm{m}^{3}$ when exposed to an exponentially time decaying field is shown in Fig. 10. Here $j_{y}$ takes an almost similar time to reach the peak value as the

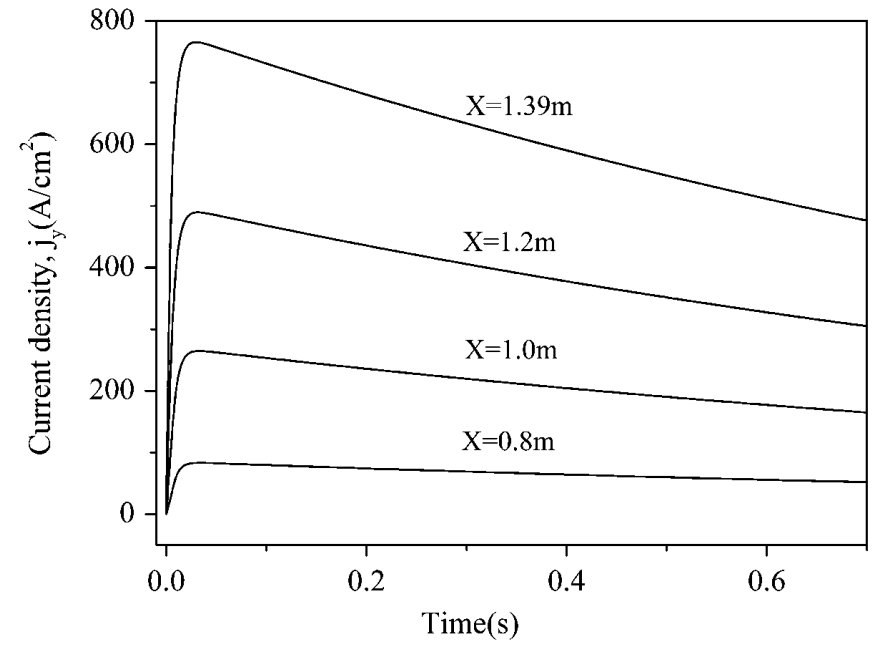

FIG. 10. Time variation of the current density at various points $(X=0.8,1.0,1.2$, and $1.39 \mathrm{~m}$ from the edge $)$ of a plate of dimension $(1.4,1.4,0.002) \mathrm{m}^{3}$ when exposed to an exponentially time decaying field. thickness of the plate is the same. We know that the value of $j_{y}$ is zero at the middle of the plate $(X=0.7 \mathrm{~m})$ and it increases as one moves towards the edge $(X=1.4 \mathrm{~m})$, as shown in Fig. 8.

For quick verification of the analytical model, the results are compared against those obtained by simulation using a finite element (FEM) based code, like OPERA [13]. The OPERA-3D analysis program ELEKTRA can be used to compute time varying electromagnetic fields in three dimensions including the effects of eddy currents. The program incorporates state of the art algorithms for the calculation of electromagnetic fields and advanced finite element numerical analysis procedures. The detailed procedure was explained in our earlier work [1]. We have taken two parallel aluminum plates of dimension $(1.4,1.4$, $0.002) \mathrm{m}^{3}$ separated by $20 \mathrm{~mm}$, inside a solenoid magnet where the field is decaying in a programmed manner as expressed in Eq. (28). The plates are placed at the center of the solenoid in such a way that the direction of the $B$ field is perpendicular to the surfaces of the plates. In other words, the normal vectors of the plate surfaces are in parallel to the rotational axis of the solenoid. The inner radius of the solenoid is $1.5 \mathrm{~m}$ and it produces a uniform field on the surfaces of the plates. The uniformity of the field is $\pm 0.05 \%$, which is similar to a dipole magnet. The mesh size of the plate is taken as $2 \times 2 \mathrm{~mm}^{2}$ in the $x-y$ plane and $0.5 \mathrm{~mm}$ along thickness $(z)$. This model is solved with $1 / 4$ symmetry and the number of elements and nodes in the model are 1378300 and 1417074 , respectively.

The values of the current density, predicted by the analytical model, are in good agreement with the simulation values obtained using a 3D FEM code [13]. The values of the eddy current density obtained from two different methods are plotted in Fig. 11 for comparison.

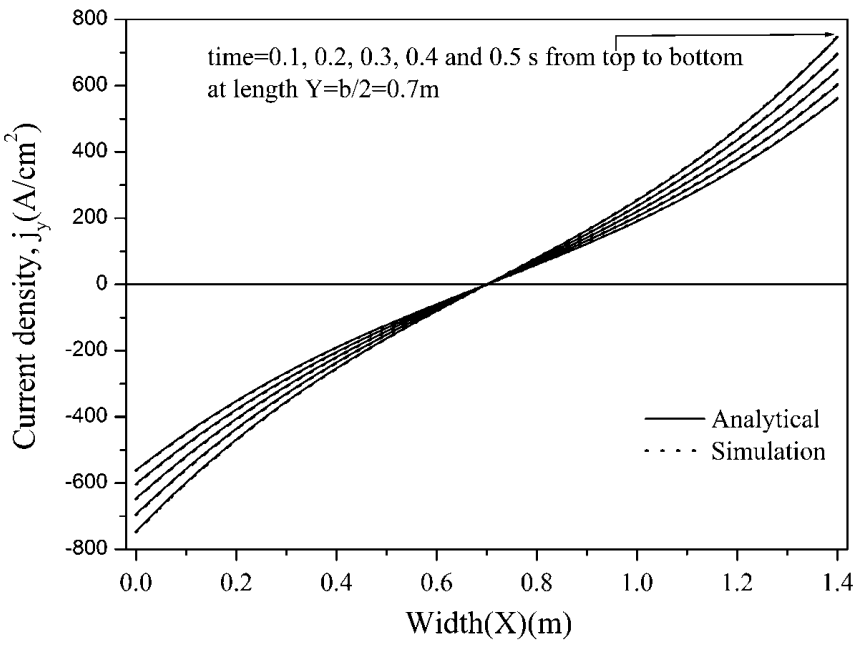

FIG. 11. Variation of the eddy current density, $j_{y}\left(\mathrm{~A} / \mathrm{cm}^{2}\right)$ in the aluminum plate of dimension $(1.4,1.4,0.002) \mathrm{m}^{3}$ at different times obtained from two different methods when exposed to an exponentially time decaying field. 
The agreements are within $0.2 \%$. This work may be extended for rectangular vacuum chambers with some approximations. A rectangular chamber is made of two such horizontal plates connected by two thin (few mm width) vertical plates at the end. Neglecting the contribution of the vertical plates, the eddy current in the chamber will be the same as that of two horizontal plates separated by a distance of $20 \mathrm{~mm}$ in this case.

The magnetic field produced by the eddy current is evaluated using Eq. (27). The $B_{Z}$ field generated due to the eddy current in the two parallel plates is plotted in Fig. 12. It shows that the field varies with the square of the distance in the central zone, a typical sextupole-like variation as expected. Here the distance is measured from the middle of the plates, i.e., at $X=a / 2=0.7 \mathrm{~m}$. However, the nature and content of the multipole depends on the distance from the plate and the point of observation. The vertical component of the field obtained from the simulation is also plotted in the same graph for comparison.

Until now we have used the dimensions of the chamber as $(1.4,1.4,0.002) \mathrm{m}^{3}$ to study the analytical model. From here on, we will be studying a chamber whose dimensions are close to the dipole magnet beam chamber used in our accelerator. We have already explained that the antechamber portion of a vacuum chamber of a storage ring remains outside the poles of a dipole magnet. Therefore, some portion of the chamber gets exposed to a uniform magnetic field produced by the dipole magnet and rest of it (antechamber part) is exposed to a space varying field of the form given in Eq. (2). The value of $\lambda$ of Eq. (2) is $0.045 \mathrm{~m}$ as obtained from the experimental data for a $50 \mathrm{~mm}$ pole gap dipole. Storage ring chambers are of different sizes and shapes. We have considered an aluminum chamber of width, length, and thickness of $0.646,2.2$, and $0.006 \mathrm{~m}$,

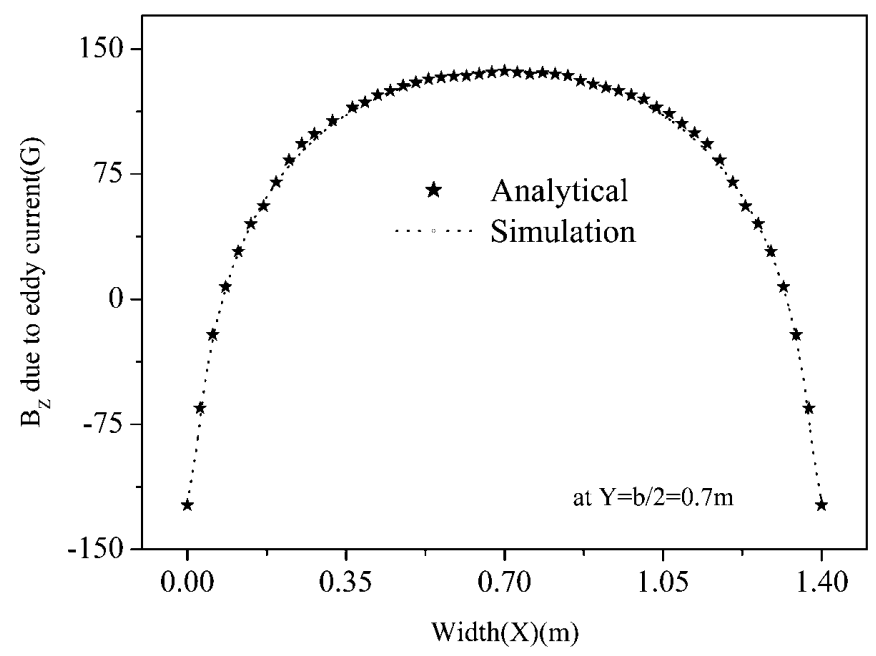

FIG. 12. Vertical component of the magnetic field, $B_{z}(G)$ due to eddy current, at the middle of the two parallel aluminum plates of dimension $(1.4,1.4,0.002) \mathrm{m}^{3} \mathrm{kept}$ at $20 \mathrm{~mm}$ apart. Simulation results (dotted lines) are also plotted for comparison. respectively. The variation of current density is studied for an arbitrary time $(t=0.1 \mathrm{~s})$ by varying the uniform field exposed zone (c) from $20 \%, 40 \%, 60 \%, 80 \%$, and $100 \%$ of the width $(a)$ of the vacuum chamber. The peak uniform field is $1.5 \mathrm{~T}$. Case II of our theoretical model can be used to study these problems. In the case of $100 \%$ uniform field exposure, the problem reduces to case I. As expected it is clear from Fig. 13 that the eddy current density is minimum at the left, passing through zero at $x=a / 2$, i.e., at $0.323 \mathrm{~m}$ and maximum at the right of the plate for $100 \%$ uniform field exposure. However, for a nonuniform field exposure the nature of the eddy current variation is asymmetric. The value of the eddy current density at the left $(X=0)$ for $20 \%$, $40 \%, 60 \%, 80 \%$, and $100 \%$ field exposure are -251.37 , $-388.55,-481.94,-532.07$, and $-543.57 \mathrm{~A} / \mathrm{cm}^{2}$, respectively, whereas at the right $(X=0.646)$, these values are $41.94,122.06,245.67,409.11$, and $543.57 \mathrm{~A} / \mathrm{cm}^{2}$, respectively.

The nature of the variation of $j_{y}$ will also depend on the width and length of the chamber. This asymmetric variation of current density causes unbalanced force acting on the chamber. Estimation of such force is important for various practical applications. The force can be evaluated using Eq. (24). We assumed that the force calculation on the plate may be extended for vacuum chambers of rectangular shape, which consists of two such horizontal plates connected by two vertical plates at the end. If we neglect the contributions coming from the vertical plates, then the force will be twice in the case of the chamber. The force acting on a chamber when exposed to a nonuniform time varying field is plotted in Fig. 14. The value of the force depends on the peak field and its extent on the chamber $(c)$. It is observed that, for a given geometry and peak field of $1.5 \mathrm{~T}$ field, the force becomes maximum when

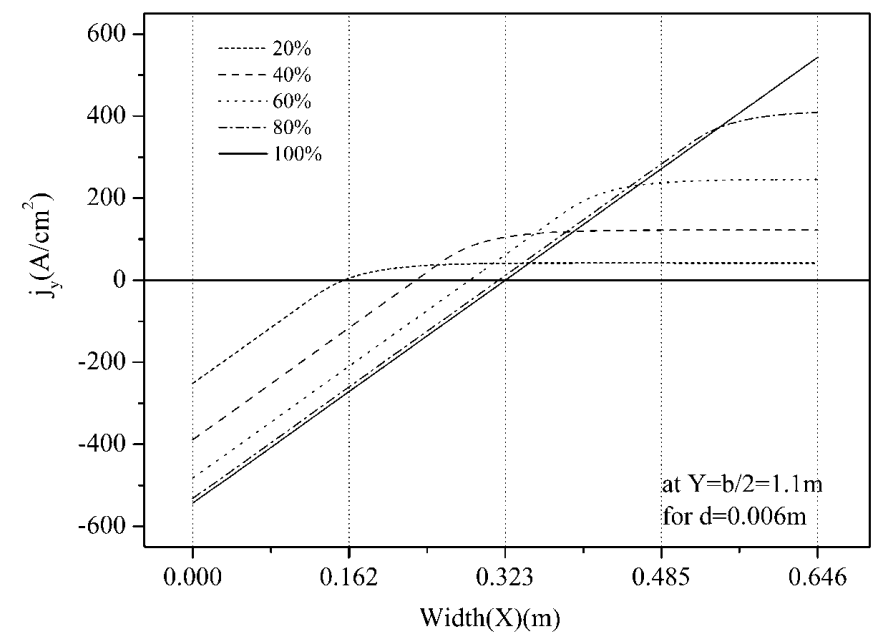

FIG. 13. Variation of the eddy current density, $j_{y}$, when the chamber is exposed to a nonuniform field as expressed in Eq. (2). The uniform field $(1.5 \mathrm{~T})$ exposure zone $(c)$ varies from $20 \%$, $40 \%, 60 \%, 80 \%$, and $100 \%$ of the width $(a=0.646 \mathrm{~m})$ of the chamber. 
approximately $60 \%$ of the chamber is exposed to a uniform field. When the extent of the uniform field zone is $100 \%$ of the chamber, then the force becomes zero (Fig. 14) as expected from the theoretical model. The value of the force is $15.2 \mathrm{kN}$ for the uniform field zone, $c$, of $0.387 \mathrm{~m}$ for a chamber of 0.646 and $2.2 \mathrm{~m}$ width and length, respectively, at time $0.1 \mathrm{~s}(d=0.006 \mathrm{~m})$. This gives general information about the force acting on the chamber of a storage ring in the case of sudden turn-off of power supply. This will be helpful in designing the antechamber part to avoid unwanted force on the chamber without compromising the vacuum consideration.

Variation of force on the chamber with time for different thickness is also studied. The wall thickness of the chamber is decided to withstand the atmospheric pressure load when the chamber is under vacuum. However, this force calculation will provide additional information about the thickness. Therefore, during the design stage, the wall thickness can be decided judiciously.

Variation of the force on a chamber with time for different wall thickness $(d=4,6,8,10$, and $14 \mathrm{~mm})$ of a chamber when exposed to a nonuniform time varying magnetic field is plotted in Fig. 15. Width and length of the chamber are 0.646 and $2.2 \mathrm{~m}$, respectively. Around $40 \%$ of the chamber $(c=0.260 \mathrm{~m})$ is exposed to $1.5 \mathrm{~T}$ peak uniform magnetic field. It is observed that the forces acting on a chamber, whose wall thickness varies from $d=4,6$, 8,10 , and $14 \mathrm{~mm}$, by keeping all other parameters unchanged, are $8.83,13.12,17.47,21.57$, and $29.66 \mathrm{kN}$, respectively, and it reaches its peak value at time $t=$ $0.038,0.042,0.046,0.053$, and $0.064 \mathrm{~s}$, respectively. Here we have assumed that the skin depth is large compared to the wall thickness.

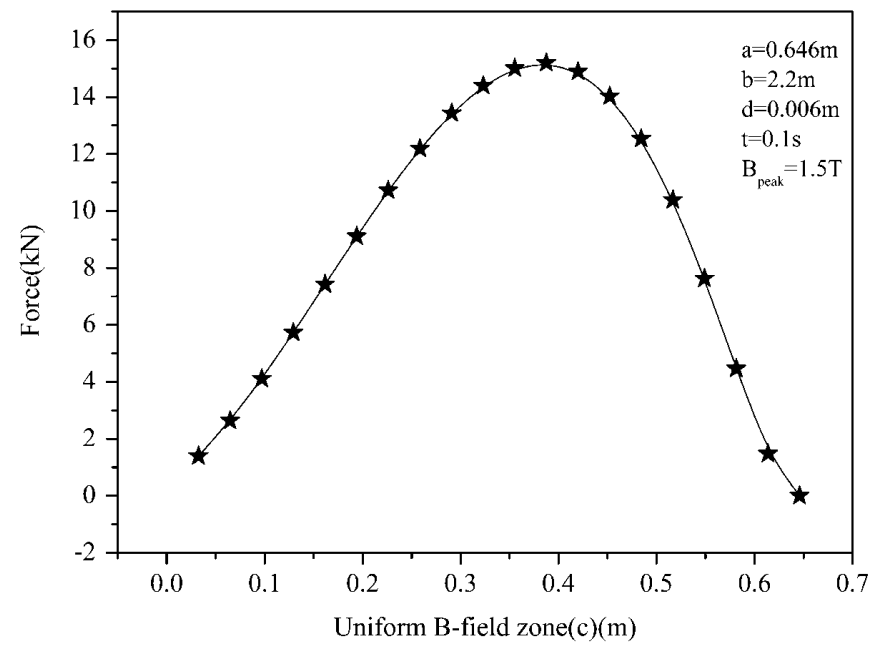

FIG. 14. The variation of the force on the chamber due to the eddy current when exposed to a nonuniform time varying magnetic field for various uniform field zones $(c)$ keeping the length and width of the plate constant as 2.2 and $0.646 \mathrm{~m}$, respectively.

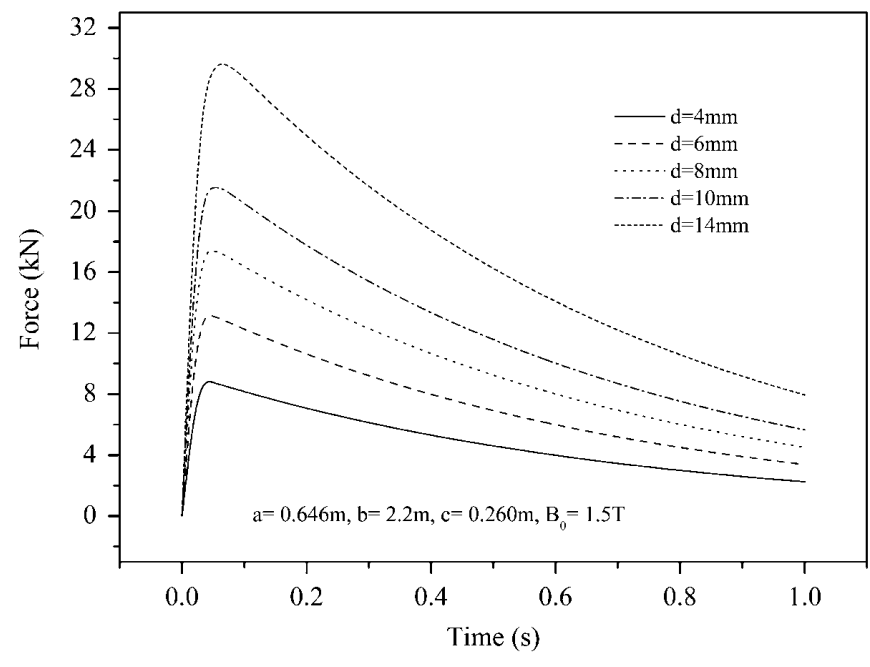

FIG. 15. Variation of the force (in $\mathrm{kN}$ ) on a chamber with time for different wall thickness $(d=4,6,8,10$, and $14 \mathrm{~mm})$ of a chamber when exposed to a nonuniform time varying magnetic field.

Our analytical model predicts that the peak force ( $F$ in $\mathrm{kN}$ ) on a chamber with the wall thickness, $d$ (in $\mathrm{mm}$ ), can be expressed as $F=2.35 d^{0.96}$. We have plotted the force variation with wall thickness of the chamber in Fig. 16 and the dotted line in the plot represents the fitted data using the above equation. However, for detailed calculation of force Eq. (24) should be used. To minimize the force on a chamber the wall thickness should be as small as possible but it should withstand the vacuum pressure. This force should be considered at the design stage of a chamber and also for providing the support structure to achieve the required stringent positional accuracy of BPMs. At the

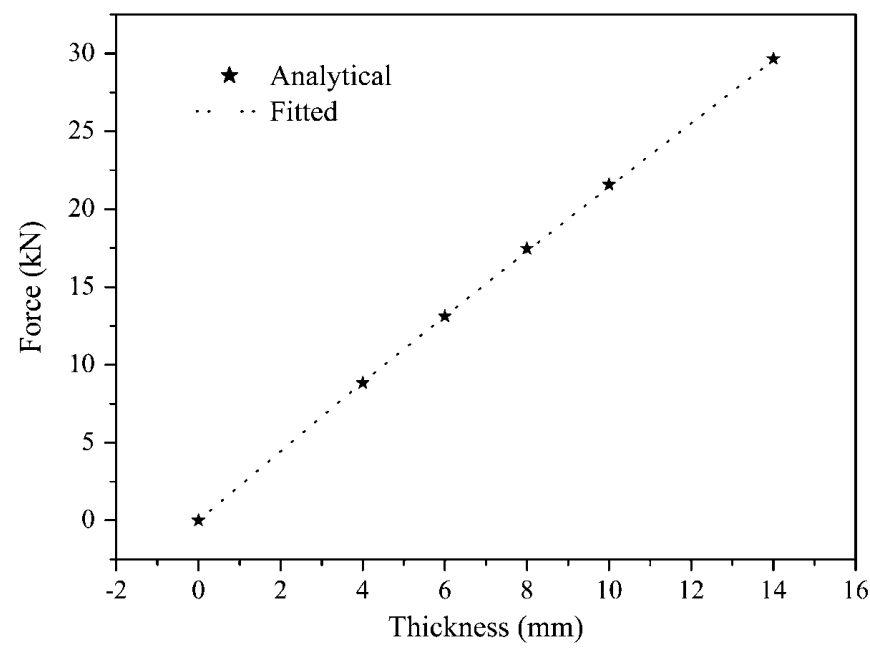

FIG. 16. Variation of the peak force (in $\mathrm{kN}$ ) on a chamber with the wall thickness of a chamber keeping other parameters constant $\left(a=0.646 \mathrm{~m}, b=2.2 \mathrm{~m}, c=0.260 \mathrm{~m}\right.$, and $B_{0}=$ $1.5 \mathrm{~T}$ ) when exposed to a nonuniform time varying magnetic field. 
same time the mechanical strength of the welding joints of a chamber should be strong enough to withstand the force acting on it when supported by a rigid structure. Our model predicts that the time of the peak force $(t$ in $\mathrm{s})$ as a function of wall thickness ( $d$ in $\mathrm{mm}$ ) can be expressed as $t=$ $0.019 d^{0.44}$ for the chamber of above dimensions.

\section{CONCLUSIONS}

The analysis presented here allows a quick and accurate estimation of the force acting on the vacuum chamber of a storage ring when exposed to a time varying magnetic field without using time consuming computer simulation. A computer code, in FORTRAN, is developed using the analytical expressions of the theoretical model that provides results of various parametric studies. This helps to understand the physical behavior of electromagnetic field better. The method has been simplified by assuming the uniform wall thickness of the chamber. A more complicated structure with varying thickness may be analyzed with modified treatment which was not considered here. Finally, the knowledge of the force on the chamber helps to design a robust chamber and its support structure. It will improve the up time of the machine and long time positional accuracy of the BPMs and, hence, the smooth operation of the ring. The force acting on a chamber due to the eddy current when exposed to a nonuniform time varying $B$ field depends on the portion of it exposed to the uniform field. When it is exposed to a uniform $B$ field, then the force acting on the one half of the chamber is balanced by the force on the other half. Therefore, the net force acting on it will be zero. It is observed that, for a given geometry and peak field, presented in this paper, the force becomes the maximum when approximately $60 \%$ of the chamber is exposed to a uniform field. This will be helpful in designing the antechamber part of the vacuum chamber to avoid unwanted force on the chamber without compromising the vacuum consideration. Variation of force on the chamber with time for different thickness is also studied. The wall thickness of the chamber is decided to withstand the atmospheric pressure load when the chamber is under vacuum. However, this force calculation will provide additional information about the wall thickness. Therefore, during the design stage, the wall thickness can be decided judiciously. Thickness should be kept as small as possible which can withstand the vacuum pressure and minimize the force due to eddy current. Variation of the eddy current density with time for different wall thickness shows interesting behavior. It takes longer time to reach the peak value for thicker chamber. It shows that for $2 \mathrm{~mm}$ wall thickness, $j_{y}$ reaches very quickly $(0.02 \mathrm{~s})$ to the peak value. However, for $18 \mathrm{~mm}$ wall thickness it takes $0.19 \mathrm{~s}$ to reach the peak value. The force on the chamber depends on the eddy current and the applied $B$ field. The $B$ field is decaying exponentially and the eddy current initially increases and then decreases with time. Therefore, the force variation shows the initial increase and then a decrease with time. It is observed that the peak force acting on a chamber of dimension $(0.646,2.2,0.014) \mathrm{m}^{3}$ is $29.66 \mathrm{kN}$ for $1.5 \mathrm{~T}$ peak field and it reaches its peak value at time $t=0.064 \mathrm{~s}$.

\section{ACKNOWLEDGMENTS}

The authors would like to acknowledge Sudhansu S. Mandal for his help and many stimulating discussions. We acknowledge Professor P. D. Gupta for useful discussion and encouragement. We would like to thank the referees for their useful comments and suggestions to improve the presentation of the paper. We acknowledge Vijendra Prasad for his help in manuscript preparation.

[1] Gautam Sinha and S.S. Prabhu, Phys. Rev. ST Accel. Beams 14, 062401 (2011).

[2] S. Y. Lee, Nucl. Instrum. Methods Phys. Res., Sect. A 300, 151 (1991).

[3] A. A. Kim, M. G. Mazarakis, V. I. Manylov, V. A. Vizir, and W. A. Stygar, Phys. Rev. ST Accel. Beams 13, 070401 (2010).

[4] Gautam Sinha, Subrat Das, S. S. Prabhu, and Gurnam Singh, in Proceedings of the 5th Indian Particle Accelerator Conference, New Delhi, India, 2011 [http:// www.iuac.res.in/InPAC2011/].

[5] Youngdo Joo, Ilmoon Hwang, Sungju Park, and Changbum Kim, Nucl. Instrum. Methods Phys. Res., Sect. A 638, 11 (2011).

[6] Haiyi Dong, Hong Song, Qi Li, Lijuan Zhou, Qi Yang, Yating Wang, and Qiong Xiao, Nucl. Instrum. Methods Phys. Res., Sect. A 620, 166 (2010).

[7] E. Al-Dmour, D. Einfeld, B. Calcagno, R. Martín, Ll. Miralles, M. Quispe, Ll. Ribó, Vacuum 82, 1441 (2008).

[8] R. Lill, G. Decker, J. Hoyt, X. Sun, and J. Wang, in Proceedings of BIW10, Santa Fe, New Mexico, 2010, TUPSM049, p. 260 [http://www.JACoW.org].

[9] L. Doom, M. Ferreira, H. C. Hseuh, F. Lincoln, C. Longo, V. Ravindranath, and S. Sharma, Report No. BNL-819012008-CP [http://www.bnl.gov/isd/documents/43871.pdf].

[10] T. Jack, Tanabe, Iron Dominated Electromagnets (World Scientific, Singapore, 2005), Chap. 7.

[11] Gautam Sinha and Gurnam Singh, Rev. Sci. Instrum. 79, 123302 (2008).

[12] C. Rathjen, in Proceedings of the 8th European Particle Accelerator Conference, Paris, France, 2002 (EPS-IGA and CERN, Geneva, 2002), p. 2580.

[13] OPERA-3D user guide, Vector Fields Limited, U.K.

[14] Multiphysics simulation software, ANSYS, Inc.

[15] G. Sinha, A. Kumar, A. K. Mishra, and G. Singh, in Proceedings of the 4th Asian Particle Accelerator Conference, Indore, India, 2007 (RRCAT, Indore, India, 2007), p. 378.

[16] G. B. Arfken and H. J. Weber, Mathematical Methods for Physicists (Academic Press, New York, 2005), 6th ed., Chap. 15. 